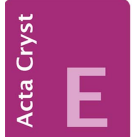

\section{Crystal structure of 1-\{2-[(2-methoxy- phenyl)selanyl]phenyl\}-4-phenyl-1H- \\ 1,2,3-triazole}

Leandro R. S. Camargo, ${ }^{a}$ Julio Zukerman-Schpector, ${ }^{a *}$ Anna M. Deobald, ${ }^{b} \neq$ Antonio L. Braga ${ }^{c}$ and Edward R. T. Tiekink $^{\mathrm{d}}$

a Departmento de Química, Universidade Federal de São Carlos, 13565-905 São Carlos, SP, Brazil, 'b Departmento de Química, Universidade Federal de Santa Maria, 97105-900 Santa Maria, RS, Brazil, `Departmento de Química, Universidade Federal de Santa Catarina, 88040-900 Florianópolis, SC, Brazil, and department of Chemistry, University of Malaya, 50603 Kuala Lumpur, Malaysia. *Correspondence e-mail: julio@power.ufscar.br

Received 12 February 2015; accepted 16 February 2015

Edited by P. C. Healy, Griffith University, Australia

In the title compound, $\mathrm{C}_{21} \mathrm{H}_{17} \mathrm{~N}_{3} \mathrm{OSe}$, the dihedral angles between the central five-membered ring and the $\mathrm{C}$ - and $\mathrm{N}$ bound rings are $17.89(10)$ and $42.35(10)^{\circ}$, respectively, indicating the molecule is twisted. The dihedral angle between the Se-bound rings is $85.36(10)^{\circ}$. A close intramolecular Se. . O contact of 2.8507 (13) $\AA$ is noted. In the crystal, C$\mathrm{H} \cdots \mathrm{O}, \mathrm{C}-\mathrm{H} \cdots \mathrm{N}$ and $\mathrm{C}-\mathrm{H} \cdots \pi$ interactions lead to the formation of supramolecular layers parallel to (011); these stack with no specific intermolecular interactions between them.

Keywords: crystal structure; organoselenium; Se $\cdots \mathrm{O}$ halogen bonding; hydrogen bonding; $\mathrm{C}-\mathrm{H} \cdots \pi$ interactions.

CCDC reference: 1049507

\section{Related literature}

For background to arylseleno-1,2,3-triazoles and to the synthesis of the title compound, see: Deobald et al. (2011). For an analysis of intra- and intermolecular Se..O interactions, see: Linden et al. (2014). For a related organoselenium compound with a 1,2,3-triazole residue, see: Camargo et al. (2015).

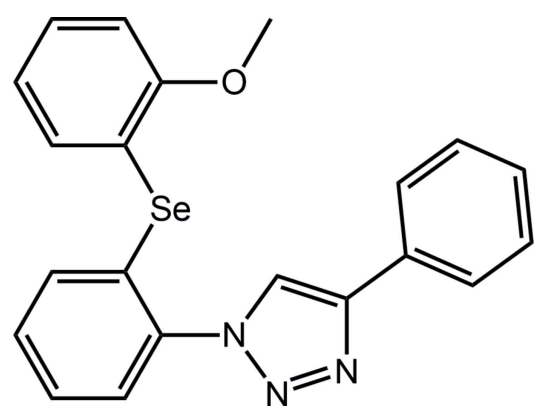

\section{Experimental}

\subsection{Crystal data}

$\mathrm{C}_{21} \mathrm{H}_{17} \mathrm{~N}_{3} \mathrm{OSe}$

$M_{r}=406.33$

Triclinic, $P \overline{1}$

$a=5.6565(3) \AA$

$b=10.3682(5) \AA$

$c=15.3358(7) \AA$

$\alpha=81.604(4)^{\circ}$

$\beta=80.006(4)^{\circ}$

\subsection{Data collection}

Agilent SuperNova CCD diffractometer

Absorption correction: multi-scan

$T_{\min }=0.759, T_{\max }=1.000$

\subsection{Refinement}

$R\left[F^{2}>2 \sigma\left(F^{2}\right)\right]=0.028$

$w R\left(F^{2}\right)=0.064$

$S=1.01$

3869 reflections

236 parameters

$\mathrm{H}$-atom parameters constrained

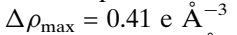

$\Delta \rho_{\min }=-0.52 \mathrm{e}^{-3}$ (CrysAlis PRO; Agilent, 2011)

Table 1

Hydrogen-bond geometry $\left(\AA,^{\circ}\right)$.

$C g 1$ is the centroid of the $\mathrm{C} 1-\mathrm{C} 6$ ring.

\begin{tabular}{llllll}
\hline$D-\mathrm{H} \cdots A$ & $D-\mathrm{H}$ & $\mathrm{H} \cdots A$ & $D \cdots A$ & \multicolumn{2}{l}{$D-\mathrm{H} \cdots A$} \\
\hline $\mathrm{C} 18-\mathrm{H} 18 \cdots \mathrm{O} 1^{\mathrm{i}}$ & 0.95 & 2.54 & $3.472(2)$ & 165 & \\
$\mathrm{C} 14-\mathrm{H} 14 \cdots \mathrm{N} 3^{\mathrm{ii}}$ & 0.95 & 2.58 & $3.520(2)$ & 170 & \\
$\mathrm{C} 10-\mathrm{H} 10 \cdots C g 1^{\text {iii }}$ & 0.95 & 2.82 & $3.630(2)$ & 144 & \\
\hline Symmetry codes: & (i) & $-x+2,-y+2,-z+1 ;$ & (ii) & $x-1, y, z ;$ & (iii) \\
$-x+1,-y+1,-z+2$. & & & & &
\end{tabular}

Data collection: CrysAlis PRO (Agilent, 2011); cell refinement: CrysAlis PRO; data reduction: CrysAlis PRO; program(s) used to solve structure: SIR2014 (Burla et al., 2015); program(s) used to refine structure: SHELXL2014 (Sheldrick, 2015); molecular graphics: ORTEP-3 for Windows (Farrugia, 2012) and DIAMOND (Brandenburg, 2006); software used to prepare material for publication: MarvinSketch (ChemAxon, 2010) and publCIF (Westrip, 2010). 


\section{Acknowledgements}

The Brazilian agencies CNPq (305626/2013-2 to JZ-S), CAPES, FAPESC and FAPESP (2010/10855-5 to LRSC) are acknowledged for financial support.

Supporting information for this paper is available from the IUCr electronic archives (Reference: HG5432).

\section{References}

Agilent (2011). CrysAlis PRO. Agilent Technologies, Yarnton, England.

Brandenburg, K. (2006). DIAMOND. Crystal Impact GbR, Bonn, Germany.

Burla, M. C., Caliandro, R., Carrozzini, B., Cascarano, G. L., Cuocci, C., Giacovazzo, C., Mallamo, M., Mazzone, A. \& Polidori, G. (2015). J. Appl. Cryst. 48, 306-309.

Camargo, L. R. S., Zukerman-Schpector, J., Deobald, A. M., Braga, A. L. \& Tiekink, E. R. T. (2015). Acta Cryst. E71, o200-o201.

ChemAxon (2010). Marvinsketch. http://www.chemaxon.com.

Deobald, A. M., Camargo, L. R. S., Hörner, M., Rodrigues, O. E. D., Alves, D. \& Braga, A. L. (2011). Synthesis, pp. 2397-2406.

Farrugia, L. J. (2012). J. Appl. Cryst. 45, 849-854.

Linden, A., Zhou, Y. \& Heimgartner, H. (2014). Acta Cryst. C70, 482-487.

Sheldrick, G. M. (2015). Acta Cryst. C71, 3-8.

Westrip, S. P. (2010). J. Appl. Cryst. 43, 920-925. 


\section{supporting information}

Acta Cryst. (2015). E71, o202-o203 [doi:10.1107/S2056989015003230]

Crystal structure of 1-\{2-[(2-methoxyphenyl)selanyl]phenyl\}-4-phenyl-1H-1,2,3triazole

\section{Leandro R. S. Camargo, Julio Zukerman-Schpector, Anna M. Deobald, Antonio L. Braga and}

\section{Edward R. T. Tiekink}

\section{S1. Experimental}

The compound was prepared in accord with the literature (Deobald et al., 2011). Crystals were obtained by taking 200 $\mathrm{mg}$ of sample into a sample vial containing methanol $(10 \mathrm{ml})$ and letting it stand at room temperature.

\section{S2. Refinement}

Carbon-bound $\mathrm{H}$-atoms were placed in calculated positions $(\mathrm{C}-\mathrm{H}=0.95$ to $0.98 \AA)$ and were included in the refinement in the riding model approximation, with $U_{i s o}(\mathrm{H})=1.2-1.5 U_{e q}(\mathrm{C})$.

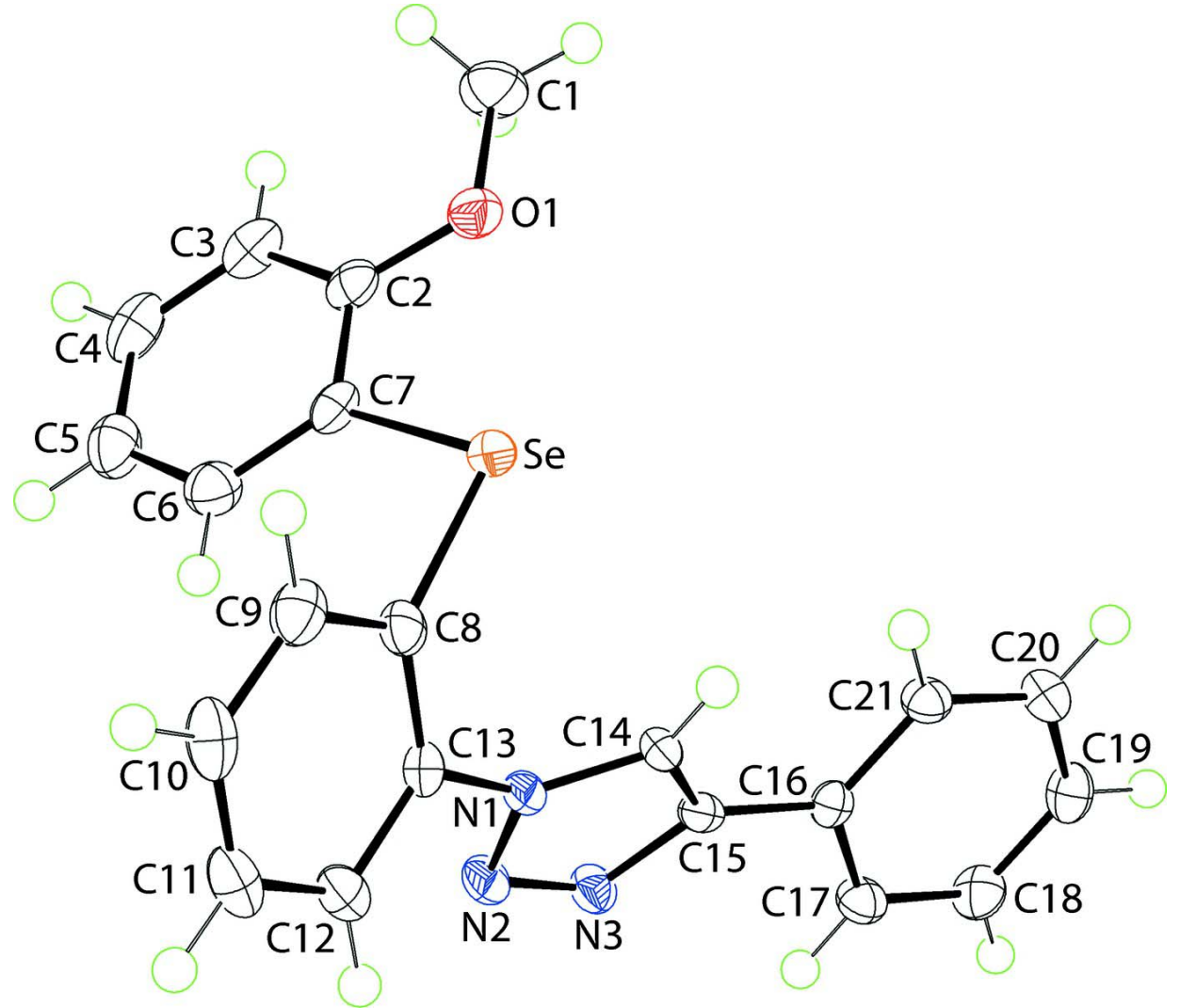

\section{Figure 1}

The molecular structure of the title compound showing the atom-labelling scheme and displacement ellipsoids at the $70 \%$ probability level. 


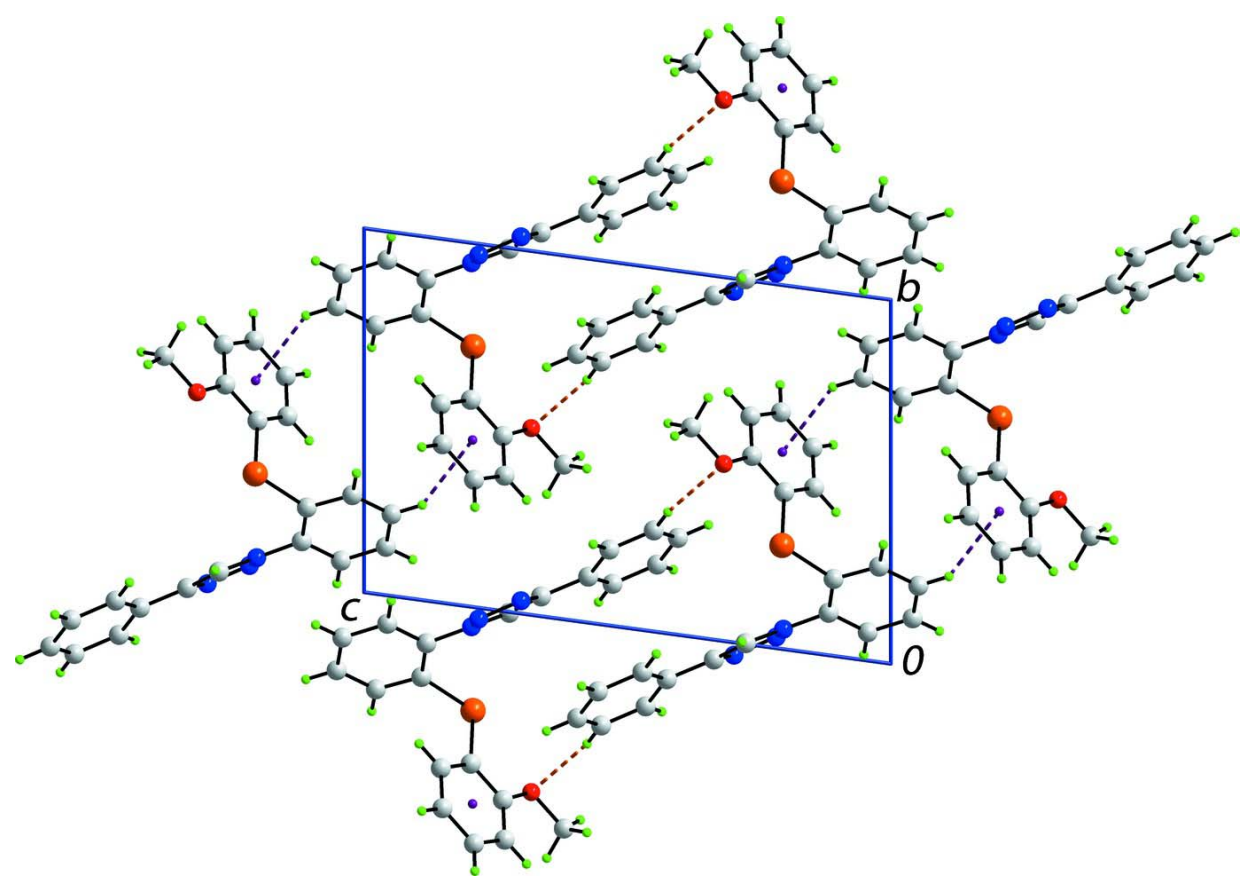

\section{Figure 2}

A view in projection down the $a$ axis of the unit-cell contents. The $\mathrm{C}-\mathrm{H} \cdots \mathrm{O}, \mathrm{C}-\mathrm{H} \cdots \mathrm{N}$ and $\mathrm{C}-\mathrm{H} \cdots \pi$ interactions are shown as orange, blue and purple dashed lines, respectively.

\section{1-\{2-[(2-Methoxyphenyl)selanyl]phenyl\}-4-phenyl-1H-1,2,3-triazole}

\section{Crystal data}

$\mathrm{C}_{21} \mathrm{H}_{17} \mathrm{~N}_{3} \mathrm{OSe}$

$M_{r}=406.33$

Triclinic, $P \overline{1}$

$a=5.6565(3) \AA$

$b=10.3682(5) \AA$

$c=15.3358$ (7) $\AA$

$\alpha=81.604(4)^{\circ}$

$\beta=80.006(4)^{\circ}$

$\gamma=85.340(4)^{\circ}$

$V=874.83(8) \AA^{3}$

\section{Data collection}

Agilent SuperNova CCD diffractometer

Radiation source: SuperNova $(\mathrm{Cu}) \mathrm{X}$-ray

Source

$\omega$ scans

Absorption correction: multi-scan

(CrysAlis PRO; Agilent, 2011)

$T_{\min }=0.759, T_{\max }=1.000$
$Z=2$

$F(000)=412$

$D_{\mathrm{x}}=1.543 \mathrm{Mg} \mathrm{m}^{-3}$

Mo $K \alpha$ radiation, $\lambda=0.71073 \AA$

Cell parameters from 4524 reflections

$\theta=2.6-29.2^{\circ}$

$\mu=2.16 \mathrm{~mm}^{-1}$

$T=100 \mathrm{~K}$

Prism, colourless

$0.30 \times 0.20 \times 0.10 \mathrm{~mm}$

6845 measured reflections

3869 independent reflections

3548 reflections with $I>2 \sigma(I)$

$R_{\text {int }}=0.040$

$\theta_{\max }=27.5^{\circ}, \theta_{\min }=2.6^{\circ}$

$h=-6 \rightarrow 7$

$k=-13 \rightarrow 12$

$l=-19 \rightarrow 19$ 


\section{Refinement}

Refinement on $F^{2}$

Least-squares matrix: full

$R\left[F^{2}>2 \sigma\left(F^{2}\right)\right]=0.028$

$w R\left(F^{2}\right)=0.064$

$S=1.01$

3869 reflections

236 parameters

0 restraints
Hydrogen site location: inferred from neighbouring sites

$\mathrm{H}$-atom parameters constrained

$w=1 /\left[\sigma^{2}\left(F_{\mathrm{o}}{ }^{2}\right)+(0.026 P)^{2}+0.4456 P\right]$

where $P=\left(F_{\mathrm{o}}^{2}+2 F_{\mathrm{c}}{ }^{2}\right) / 3$

$(\Delta / \sigma)_{\max }=0.002$

$\Delta \rho_{\max }=0.41$ e $\AA^{-3}$

$\Delta \rho_{\min }=-0.52$ e $\AA^{-3}$

Special details

Geometry. All e.s.d.'s (except the e.s.d. in the dihedral angle between two l.s. planes) are estimated using the full covariance matrix. The cell e.s.d.'s are taken into account individually in the estimation of e.s.d.'s in distances, angles and torsion angles; correlations between e.s.d.'s in cell parameters are only used when they are defined by crystal symmetry. An approximate (isotropic) treatment of cell e.s.d.'s is used for estimating e.s.d.'s involving 1.s. planes.

Fractional atomic coordinates and isotropic or equivalent isotropic displacement parameters $\left(\AA^{2}\right)$

\begin{tabular}{|c|c|c|c|c|}
\hline & $x$ & $y$ & $z$ & $U_{\text {iso }} * / U_{\text {eq }}$ \\
\hline $\mathrm{Se}$ & $0.46182(3)$ & $0.71551(2)$ & $0.79326(2)$ & $0.01503(7)$ \\
\hline $\mathrm{O} 1$ & $0.5130(2)$ & $0.51326(12)$ & $0.68268(9)$ & $0.0185(3)$ \\
\hline N1 & $0.8216(3)$ & $0.94528(14)$ & $0.79605(10)$ & 0.0128 \\
\hline N2 & $1.0599(3)$ & $0.96928(15)$ & $0.78243(11)$ & $0.0154(3)$ \\
\hline N3 & $1.1085(3)$ & $1.03509(15)$ & $0.70214(10)$ & 0.0150 \\
\hline $\mathrm{C} 1$ & $0.4930(4)$ & $0.4251(2)$ & $0.62080(14)$ & $0.0255(5)$ \\
\hline $\mathrm{H} 1 \mathrm{~A}$ & 0.3570 & 0.4549 & 0.5897 & $0.038^{*}$ \\
\hline H1B & 0.4669 & 0.3374 & 0.6533 & $0.038^{*}$ \\
\hline $\mathrm{H} 1 \mathrm{C}$ & 0.6414 & 0.4227 & 0.5771 & $0.038^{*}$ \\
\hline $\mathrm{C} 2$ & 0.6840 & $0.48102(18)$ & $0.73647(12)$ & $0.0164(4)$ \\
\hline $\mathrm{C} 3$ & $0.8446(4)$ & $0.37256(18)$ & $0.73332(13)$ & $0.0209(4)$ \\
\hline H3 & 0.8404 & 0.3139 & 0.6916 & $0.025^{*}$ \\
\hline $\mathrm{C} 4$ & $1.0113(4)$ & $0.34994(19)$ & $0.79132(14)$ & 0.0238 \\
\hline H4 & 1.1196 & 0.2750 & 0.7897 & $0.029 *$ \\
\hline $\mathrm{C} 5$ & $1.0204(4)$ & $0.4358(2)$ & $0.85130(14)$ & $0.0227(4)$ \\
\hline H5 & 1.1355 & 0.4202 & 0.8905 & $0.027^{*}$ \\
\hline C6 & $0.8611(3)$ & 0.54507 (19) & $0.85434(13)$ & $0.0194(4)$ \\
\hline H6 & 0.8684 & 0.6041 & 0.8956 & $0.023 *$ \\
\hline $\mathrm{C} 7$ & $0.6917(3)$ & $0.56852(17)$ & $0.79755(12)$ & $0.0150(4)$ \\
\hline $\mathrm{C} 8$ & $0.5446(3)$ & $0.78849(18)$ & $0.89132(12)$ & 0.0153 \\
\hline C9 & $0.4431(4)$ & $0.73766(19)$ & $0.97760(13)$ & $0.0194(4)$ \\
\hline H9 & 0.3236 & 0.6758 & 0.9856 & $0.023^{*}$ \\
\hline $\mathrm{C} 10$ & $0.5126(4)$ & $0.7755(2)$ & $1.05209(13)$ & $0.0223(4)$ \\
\hline $\mathrm{H} 10$ & 0.4427 & 0.7386 & 1.1104 & $0.027 *$ \\
\hline C11 & $0.6845(4)$ & $0.8671(2)$ & $1.04128(13)$ & $0.0224(4)$ \\
\hline H11 & 0.7347 & 0.8922 & 1.0920 & $0.027^{*}$ \\
\hline $\mathrm{C} 12$ & $0.7825(4)$ & 0.92197 (19) & $0.95603(13)$ & $0.0182(4)$ \\
\hline H12 & 0.8974 & 0.9863 & 0.9485 & $0.022 *$ \\
\hline C13 & $0.7135(3)$ & $0.88317(17)$ & $0.88174(12)$ & $0.0133(4)$ \\
\hline C14 & 0.7201 & $0.99764(17)$ & $0.72442(12)$ & $0.0127(3)$ \\
\hline
\end{tabular}




\begin{tabular}{lllll}
$\mathrm{H} 14$ & 0.5569 & 0.9958 & 0.7172 & $0.015^{*}$ \\
$\mathrm{C} 15$ & $0.9046(3)$ & $1.05438(17)$ & $0.66405(12)$ & $0.0124(3)$ \\
$\mathrm{C} 16$ & $0.8998(3)$ & $1.12626(17)$ & $0.57456(12)$ & $0.0126(4)$ \\
$\mathrm{C} 17$ & $1.0842(3)$ & $1.20866(18)$ & $0.53447(13)$ & $0.0166(4)$ \\
$\mathrm{H} 17$ & 1.2131 & 1.2173 & 0.5651 & $0.020^{*}$ \\
$\mathrm{C} 18$ & $1.0791(3)$ & $1.27779(19)$ & $0.44998(13)$ & $0.0192(4)$ \\
$\mathrm{H} 18$ & 1.2047 & 1.3334 & 0.4233 & $0.023^{*}$ \\
$\mathrm{C} 19$ & $0.8925(3)$ & $1.26635(19)$ & $0.40436(13)$ & $0.0184(4)$ \\
$\mathrm{H} 19$ & 0.8903 & 1.3136 & 0.3465 & $0.022^{*}$ \\
$\mathrm{C} 20$ & $0.7089(3)$ & $1.18527(18)$ & $0.44379(13)$ & $0.0180(4)$ \\
$\mathrm{H} 20$ & 0.5802 & 1.1772 & 0.4129 & $0.022^{*}$ \\
$\mathrm{C} 21$ & $0.7127(3)$ & $1.11581(18)$ & $0.52828(12)$ & $0.0152(4)$ \\
$\mathrm{H} 21$ & 0.5861 & 1.0606 & 0.5547 & $0.018^{*}$ \\
\hline
\end{tabular}

Atomic displacement parameters $\left(\AA^{2}\right)$

\begin{tabular}{lllllll}
\hline & $U^{11}$ & $U^{22}$ & $U^{33}$ & $U^{12}$ & $U^{13}$ & $U^{23}$ \\
\hline $\mathrm{Se}$ & $0.01551(10)$ & $0.01235(10)$ & $0.01832(11)$ & $-0.00120(7)$ & $-0.00576(7)$ & $-0.00192(7)$ \\
$\mathrm{O} 1$ & $0.0237(7)$ & $0.0152(7)$ & $0.0177(7)$ & $-0.0024(5)$ & $-0.0049(6)$ & $-0.0032(5)$ \\
$\mathrm{N} 1$ & $0.0117(7)$ & $0.0131(7)$ & $0.0145(8)$ & $-0.0021(6)$ & $-0.0051(6)$ & $-0.0002(6)$ \\
$\mathrm{N} 2$ & $0.0108(7)$ & $0.0180(8)$ & $0.0185(8)$ & $-0.0012(6)$ & $-0.0056(6)$ & $-0.0015(6)$ \\
$\mathrm{N} 3$ & $0.0125(7)$ & $0.0174(8)$ & $0.0155(8)$ & $-0.0012(6)$ & $-0.0040(6)$ & $-0.0011(6)$ \\
$\mathrm{C} 1$ & $0.0338(12)$ & $0.0244(11)$ & $0.0210(10)$ & $-0.0045(9)$ & $-0.0057(9)$ & $-0.0090(8)$ \\
$\mathrm{C} 2$ & $0.0193(9)$ & $0.0138(9)$ & $0.0147(9)$ & $-0.0045(7)$ & $0.0008(8)$ & $0.0010(7)$ \\
$\mathrm{C} 3$ & $0.0252(10)$ & $0.0148(9)$ & $0.0200(10)$ & $-0.0012(8)$ & $0.0030(8)$ & $-0.0017(8)$ \\
$\mathrm{C} 4$ & $0.0218(10)$ & $0.0165(10)$ & $0.0282(11)$ & $0.0032(8)$ & $0.0033(9)$ & $0.0028(8)$ \\
$\mathrm{C} 5$ & $0.0182(10)$ & $0.0219(10)$ & $0.0265(11)$ & $0.0008(8)$ & $-0.0053(9)$ & $0.0021(8)$ \\
$\mathrm{C} 6$ & $0.0182(9)$ & $0.0181(9)$ & $0.0218(10)$ & $-0.0020(7)$ & $-0.0031(8)$ & $-0.0024(8)$ \\
$\mathrm{C} 7$ & $0.0148(9)$ & $0.0112(8)$ & $0.0168(9)$ & $-0.0014(7)$ & $0.0006(7)$ & $0.0014(7)$ \\
$\mathrm{C} 8$ & $0.0142(9)$ & $0.0152(9)$ & $0.0168(9)$ & $0.0021(7)$ & $-0.0053(7)$ & $-0.0016(7)$ \\
$\mathrm{C} 9$ & $0.0177(9)$ & $0.0185(10)$ & $0.0200(10)$ & $-0.0012(7)$ & $-0.0018(8)$ & $0.0020(8)$ \\
$\mathrm{C} 10$ & $0.0228(10)$ & $0.0273(11)$ & $0.0139(9)$ & $0.0043(8)$ & $-0.0026(8)$ & $0.0031(8)$ \\
$\mathrm{C} 11$ & $0.0243(10)$ & $0.0285(11)$ & $0.0154(10)$ & $0.0017(8)$ & $-0.0081(8)$ & $-0.0020(8)$ \\
$\mathrm{C} 12$ & $0.0178(9)$ & $0.0205(10)$ & $0.0179(10)$ & $-0.0017(7)$ & $-0.0074(8)$ & $-0.0017(8)$ \\
$\mathrm{C} 13$ & $0.0129(8)$ & $0.0135(8)$ & $0.0126(9)$ & $0.0018(7)$ & $-0.0029(7)$ & $0.0006(7)$ \\
$\mathrm{C} 14$ & $0.0129(8)$ & $0.0135(8)$ & $0.0129(9)$ & $-0.0002(7)$ & $-0.0056(7)$ & $-0.0021(7)$ \\
$\mathrm{C} 15$ & $0.0110(8)$ & $0.0123(8)$ & $0.0148(9)$ & $-0.0003(6)$ & $-0.0031(7)$ & $-0.0043(7)$ \\
$\mathrm{C} 16$ & $0.0122(8)$ & $0.0121(8)$ & $0.0127(9)$ & $0.0015(7)$ & $-0.0007(7)$ & $-0.0022(7)$ \\
$\mathrm{C} 17$ & $0.0139(9)$ & $0.0183(9)$ & $0.0183(10)$ & $-0.0031(7)$ & $-0.0036(8)$ & $-0.0022(7)$ \\
$\mathrm{C} 18$ & $0.0178(9)$ & $0.0187(9)$ & $0.0191(10)$ & $-0.0047(7)$ & $0.0002(8)$ & $0.0018(8)$ \\
$\mathrm{C} 19$ & $0.0199(10)$ & $0.0193(9)$ & $0.0136(9)$ & $0.0020(8)$ & $0.0000(8)$ & $0.0012(7)$ \\
$\mathrm{C} 20$ & $0.0168(9)$ & $0.0208(10)$ & $0.0174(10)$ & $-0.0006(8)$ & $-0.0060(8)$ & $-0.0021(8)$ \\
$\mathrm{C} 21$ & $0.0134(9)$ & $0.0160(9)$ & $0.0163(9)$ & $-0.0032(7)$ & $-0.0023(7)$ & $-0.0018(7)$ \\
& & & & & & \\
\hline
\end{tabular}

Geometric parameters $\left(\AA,{ }^{\circ}\right)$

\begin{tabular}{llll}
\hline $\mathrm{Se}-\mathrm{C} 8$ & $1.9202(19)$ & $\mathrm{C} 8-\mathrm{C} 13$ & $1.400(2)$ \\
$\mathrm{Se}-\mathrm{C} 7$ & $1.9224(19)$ & $\mathrm{C} 9-\mathrm{C} 10$ & $1.388(3)$
\end{tabular}




\begin{tabular}{|c|c|c|c|}
\hline $\mathrm{O} 1-\mathrm{C} 2$ & $1.368(2)$ & C9--H9 & 0.9500 \\
\hline $\mathrm{O} 1-\mathrm{C} 1$ & $1.434(2)$ & $\mathrm{C} 10-\mathrm{C} 11$ & $1.387(3)$ \\
\hline $\mathrm{N} 1-\mathrm{C} 14$ & $1.351(2)$ & $\mathrm{C} 10-\mathrm{H} 10$ & 0.9500 \\
\hline $\mathrm{N} 1-\mathrm{N} 2$ & $1.365(2)$ & $\mathrm{C} 11-\mathrm{C} 12$ & $1.387(3)$ \\
\hline $\mathrm{N} 1-\mathrm{C} 13$ & $1.433(2)$ & $\mathrm{C} 11-\mathrm{H} 11$ & 0.9500 \\
\hline $\mathrm{N} 2-\mathrm{N} 3$ & $1.313(2)$ & $\mathrm{C} 12-\mathrm{C} 13$ & $1.388(3)$ \\
\hline N3-C15 & $1.369(2)$ & $\mathrm{C} 12-\mathrm{H} 12$ & 0.9500 \\
\hline $\mathrm{C} 1-\mathrm{H} 1 \mathrm{~A}$ & 0.9800 & $\mathrm{C} 14-\mathrm{C} 15$ & $1.378(2)$ \\
\hline $\mathrm{C} 1-\mathrm{H} 1 \mathrm{~B}$ & 0.9800 & C14-H14 & 0.9500 \\
\hline $\mathrm{C} 1-\mathrm{H} 1 \mathrm{C}$ & 0.9800 & $\mathrm{C} 15-\mathrm{C} 16$ & $1.467(2)$ \\
\hline $\mathrm{C} 2-\mathrm{C} 3$ & $1.388(3)$ & $\mathrm{C} 16-\mathrm{C} 21$ & $1.392(3)$ \\
\hline $\mathrm{C} 2-\mathrm{C} 7$ & $1.404(3)$ & $\mathrm{C} 16-\mathrm{C} 17$ & $1.402(2)$ \\
\hline $\mathrm{C} 3-\mathrm{C} 4$ & $1.389(3)$ & $\mathrm{C} 17-\mathrm{C} 18$ & $1.390(3)$ \\
\hline $\mathrm{C} 3-\mathrm{H} 3$ & 0.9500 & C17-H17 & 0.9500 \\
\hline $\mathrm{C} 4-\mathrm{C} 5$ & $1.380(3)$ & $\mathrm{C} 18-\mathrm{C} 19$ & $1.385(3)$ \\
\hline $\mathrm{C} 4-\mathrm{H} 4$ & 0.9500 & $\mathrm{C} 18-\mathrm{H} 18$ & 0.9500 \\
\hline $\mathrm{C} 5-\mathrm{C} 6$ & $1.390(3)$ & $\mathrm{C} 19-\mathrm{C} 20$ & $1.388(3)$ \\
\hline $\mathrm{C} 5-\mathrm{H} 5$ & 0.9500 & C19-H19 & 0.9500 \\
\hline $\mathrm{C} 6-\mathrm{C} 7$ & $1.388(3)$ & $\mathrm{C} 20-\mathrm{C} 21$ & $1.390(3)$ \\
\hline $\mathrm{C} 6-\mathrm{H} 6$ & 0.9500 & $\mathrm{C} 20-\mathrm{H} 20$ & 0.9500 \\
\hline $\mathrm{C} 8-\mathrm{C} 9$ & $1.393(3)$ & $\mathrm{C} 21-\mathrm{H} 21$ & 0.9500 \\
\hline $\mathrm{C} 8-\mathrm{Se}-\mathrm{C} 7$ & $96.32(8)$ & $\mathrm{C} 11-\mathrm{C} 10-\mathrm{C} 9$ & $119.82(18)$ \\
\hline $\mathrm{C} 2-\mathrm{O} 1-\mathrm{C} 1$ & $117.14(16)$ & $\mathrm{C} 11-\mathrm{C} 10-\mathrm{H} 10$ & 120.1 \\
\hline $\mathrm{C} 14-\mathrm{N} 1-\mathrm{N} 2$ & $110.78(14)$ & $\mathrm{C} 9-\mathrm{C} 10-\mathrm{H} 10$ & 120.1 \\
\hline $\mathrm{C} 14-\mathrm{N} 1-\mathrm{C} 13$ & $130.06(15)$ & $\mathrm{C} 12-\mathrm{C} 11-\mathrm{C} 10$ & $119.69(19)$ \\
\hline $\mathrm{N} 2-\mathrm{N} 1-\mathrm{C} 13$ & $118.87(15)$ & $\mathrm{C} 12-\mathrm{C} 11-\mathrm{H} 11$ & 120.2 \\
\hline $\mathrm{N} 3-\mathrm{N} 2-\mathrm{N} 1$ & $106.67(14)$ & $\mathrm{C} 10-\mathrm{C} 11-\mathrm{H} 11$ & 120.2 \\
\hline $\mathrm{N} 2-\mathrm{N} 3-\mathrm{C} 15$ & $109.61(14)$ & $\mathrm{C} 11-\mathrm{C} 12-\mathrm{C} 13$ & $120.24(18)$ \\
\hline $\mathrm{O} 1-\mathrm{C} 1-\mathrm{H} 1 \mathrm{~A}$ & 109.5 & $\mathrm{C} 11-\mathrm{C} 12-\mathrm{H} 12$ & 119.9 \\
\hline $\mathrm{O} 1-\mathrm{C} 1-\mathrm{H} 1 \mathrm{~B}$ & 109.5 & $\mathrm{C} 13-\mathrm{C} 12-\mathrm{H} 12$ & 119.9 \\
\hline $\mathrm{H} 1 \mathrm{~A}-\mathrm{C} 1-\mathrm{H} 1 \mathrm{~B}$ & 109.5 & $\mathrm{C} 12-\mathrm{C} 13-\mathrm{C} 8$ & $120.86(16)$ \\
\hline $\mathrm{O} 1-\mathrm{C} 1-\mathrm{H} 1 \mathrm{C}$ & 109.5 & $\mathrm{C} 12-\mathrm{C} 13-\mathrm{N} 1$ & $116.82(16)$ \\
\hline $\mathrm{H} 1 \mathrm{~A}-\mathrm{C} 1-\mathrm{H} 1 \mathrm{C}$ & 109.5 & $\mathrm{C} 8-\mathrm{C} 13-\mathrm{N} 1$ & $122.32(16)$ \\
\hline $\mathrm{H} 1 \mathrm{~B}-\mathrm{C} 1-\mathrm{H} 1 \mathrm{C}$ & 109.5 & $\mathrm{~N} 1-\mathrm{C} 14-\mathrm{C} 15$ & $104.97(16)$ \\
\hline $\mathrm{O} 1-\mathrm{C} 2-\mathrm{C} 3$ & $125.05(17)$ & $\mathrm{N} 1-\mathrm{C} 14-\mathrm{H} 14$ & 127.5 \\
\hline $\mathrm{O} 1-\mathrm{C} 2-\mathrm{C} 7$ & $114.90(17)$ & $\mathrm{C} 15-\mathrm{C} 14-\mathrm{H} 14$ & 127.5 \\
\hline $\mathrm{C} 3-\mathrm{C} 2-\mathrm{C} 7$ & 120.05 (19) & $\mathrm{N} 3-\mathrm{C} 15-\mathrm{C} 14$ & $107.96(16)$ \\
\hline $\mathrm{C} 2-\mathrm{C} 3-\mathrm{C} 4$ & $119.93(19)$ & $\mathrm{N} 3-\mathrm{C} 15-\mathrm{C} 16$ & $122.74(15)$ \\
\hline $\mathrm{C} 2-\mathrm{C} 3-\mathrm{H} 3$ & 120.0 & $\mathrm{C} 14-\mathrm{C} 15-\mathrm{C} 16$ & $129.29(17)$ \\
\hline $\mathrm{C} 4-\mathrm{C} 3-\mathrm{H} 3$ & 120.0 & $\mathrm{C} 21-\mathrm{C} 16-\mathrm{C} 17$ & $118.71(17)$ \\
\hline $\mathrm{C} 5-\mathrm{C} 4-\mathrm{C} 3$ & $120.32(19)$ & $\mathrm{C} 21-\mathrm{C} 16-\mathrm{C} 15$ & $121.23(16)$ \\
\hline $\mathrm{C} 5-\mathrm{C} 4-\mathrm{H} 4$ & 119.8 & $\mathrm{C} 17-\mathrm{C} 16-\mathrm{C} 15$ & $120.06(17)$ \\
\hline $\mathrm{C} 3-\mathrm{C} 4-\mathrm{H} 4$ & 119.8 & $\mathrm{C} 18-\mathrm{C} 17-\mathrm{C} 16$ & $120.19(18)$ \\
\hline $\mathrm{C} 4-\mathrm{C} 5-\mathrm{C} 6$ & $120.0(2)$ & $\mathrm{C} 18-\mathrm{C} 17-\mathrm{H} 17$ & 119.9 \\
\hline $\mathrm{C} 4-\mathrm{C} 5-\mathrm{H} 5$ & 120.0 & $\mathrm{C} 16-\mathrm{C} 17-\mathrm{H} 17$ & 119.9 \\
\hline $\mathrm{C} 6-\mathrm{C} 5-\mathrm{H} 5$ & 120.0 & $\mathrm{C} 19-\mathrm{C} 18-\mathrm{C} 17$ & $120.64(17)$ \\
\hline $\mathrm{C} 7-\mathrm{C} 6-\mathrm{C} 5$ & $120.45(19)$ & $\mathrm{C} 19-\mathrm{C} 18-\mathrm{H} 18$ & 119.7 \\
\hline
\end{tabular}




\begin{tabular}{|c|c|c|c|}
\hline $\mathrm{C} 7-\mathrm{C} 6-\mathrm{H} 6$ & 119.8 & $\mathrm{C} 17-\mathrm{C} 18-\mathrm{H} 18$ & 119.7 \\
\hline $\mathrm{C} 5-\mathrm{C} 6-\mathrm{H} 6$ & 119.8 & $\mathrm{C} 18-\mathrm{C} 19-\mathrm{C} 20$ & $119.46(18)$ \\
\hline $\mathrm{C} 6-\mathrm{C} 7-\mathrm{C} 2$ & $119.22(18)$ & $\mathrm{C} 18-\mathrm{C} 19-\mathrm{H} 19$ & 120.3 \\
\hline $\mathrm{C} 6-\mathrm{C} 7-\mathrm{Se}$ & $125.27(14)$ & $\mathrm{C} 20-\mathrm{C} 19-\mathrm{H} 19$ & 120.3 \\
\hline $\mathrm{C} 2-\mathrm{C} 7-\mathrm{Se}$ & $115.50(15)$ & $\mathrm{C} 19-\mathrm{C} 20-\mathrm{C} 21$ & $120.26(18)$ \\
\hline $\mathrm{C} 9-\mathrm{C} 8-\mathrm{C} 13$ & $117.89(17)$ & $\mathrm{C} 19-\mathrm{C} 20-\mathrm{H} 20$ & 119.9 \\
\hline $\mathrm{C} 9-\mathrm{C} 8-\mathrm{Se}$ & $117.98(14)$ & $\mathrm{C} 21-\mathrm{C} 20-\mathrm{H} 20$ & 119.9 \\
\hline $\mathrm{C} 13-\mathrm{C} 8-\mathrm{Se}$ & $123.97(14)$ & $\mathrm{C} 20-\mathrm{C} 21-\mathrm{C} 16$ & $120.73(17)$ \\
\hline $\mathrm{C} 10-\mathrm{C} 9-\mathrm{C} 8$ & $121.44(18)$ & $\mathrm{C} 20-\mathrm{C} 21-\mathrm{H} 21$ & 119.6 \\
\hline $\mathrm{C} 10-\mathrm{C} 9-\mathrm{H} 9$ & 119.3 & $\mathrm{C} 16-\mathrm{C} 21-\mathrm{H} 21$ & 119.6 \\
\hline $\mathrm{C} 8-\mathrm{C} 9-\mathrm{H} 9$ & 119.3 & & \\
\hline $\mathrm{C} 14-\mathrm{N} 1-\mathrm{N} 2-\mathrm{N} 3$ & $-0.75(19)$ & $\mathrm{C} 9-\mathrm{C} 8-\mathrm{C} 13-\mathrm{N} 1$ & $-177.53(17)$ \\
\hline $\mathrm{C} 13-\mathrm{N} 1-\mathrm{N} 2-\mathrm{N} 3$ & $-175.16(14)$ & $\mathrm{Se}-\mathrm{C} 8-\mathrm{C} 13-\mathrm{N} 1$ & $7.1(3)$ \\
\hline $\mathrm{N} 1-\mathrm{N} 2-\mathrm{N} 3-\mathrm{C} 15$ & $0.31(19)$ & $\mathrm{C} 14-\mathrm{N} 1-\mathrm{C} 13-\mathrm{C} 12$ & $-133.19(19)$ \\
\hline $\mathrm{C} 1-\mathrm{O} 1-\mathrm{C} 2-\mathrm{C} 3$ & $2.6(3)$ & $\mathrm{N} 2-\mathrm{N} 1-\mathrm{C} 13-\mathrm{C} 12$ & $40.0(2)$ \\
\hline $\mathrm{C} 1-\mathrm{O} 1-\mathrm{C} 2-\mathrm{C} 7$ & $-178.26(16)$ & $\mathrm{C} 14-\mathrm{N} 1-\mathrm{C} 13-\mathrm{C} 8$ & $46.3(3)$ \\
\hline $\mathrm{O} 1-\mathrm{C} 2-\mathrm{C} 3-\mathrm{C} 4$ & $179.81(17)$ & $\mathrm{N} 2-\mathrm{N} 1-\mathrm{C} 13-\mathrm{C} 8$ & $-140.54(18)$ \\
\hline $\mathrm{C} 7-\mathrm{C} 2-\mathrm{C} 3-\mathrm{C} 4$ & $0.7(3)$ & $\mathrm{N} 2-\mathrm{N} 1-\mathrm{C} 14-\mathrm{C} 15$ & $0.86(19)$ \\
\hline $\mathrm{C} 2-\mathrm{C} 3-\mathrm{C} 4-\mathrm{C} 5$ & $-0.9(3)$ & $\mathrm{C} 13-\mathrm{N} 1-\mathrm{C} 14-\mathrm{C} 15$ & $174.46(17)$ \\
\hline $\mathrm{C} 3-\mathrm{C} 4-\mathrm{C} 5-\mathrm{C} 6$ & $0.4(3)$ & $\mathrm{N} 2-\mathrm{N} 3-\mathrm{C} 15-\mathrm{C} 14$ & $0.2(2)$ \\
\hline $\mathrm{C} 4-\mathrm{C} 5-\mathrm{C} 6-\mathrm{C} 7$ & $0.2(3)$ & $\mathrm{N} 2-\mathrm{N} 3-\mathrm{C} 15-\mathrm{C} 16$ & $179.29(16)$ \\
\hline $\mathrm{C} 5-\mathrm{C} 6-\mathrm{C} 7-\mathrm{C} 2$ & $-0.5(3)$ & $\mathrm{N} 1-\mathrm{C} 14-\mathrm{C} 15-\mathrm{N} 3$ & $-0.65(19)$ \\
\hline $\mathrm{C} 5-\mathrm{C} 6-\mathrm{C} 7-\mathrm{Se}$ & $-179.52(14)$ & $\mathrm{N} 1-\mathrm{C} 14-\mathrm{C} 15-\mathrm{C} 16$ & $-179.64(17)$ \\
\hline $\mathrm{O} 1-\mathrm{C} 2-\mathrm{C} 7-\mathrm{C} 6$ & $-179.22(16)$ & $\mathrm{N} 3-\mathrm{C} 15-\mathrm{C} 16-\mathrm{C} 21$ & $162.95(17)$ \\
\hline $\mathrm{C} 3-\mathrm{C} 2-\mathrm{C} 7-\mathrm{C} 6$ & $0.0(3)$ & $\mathrm{C} 14-\mathrm{C} 15-\mathrm{C} 16-\mathrm{C} 21$ & $-18.2(3)$ \\
\hline $\mathrm{O} 1-\mathrm{C} 2-\mathrm{C} 7-\mathrm{Se}$ & $-0.1(2)$ & $\mathrm{N} 3-\mathrm{C} 15-\mathrm{C} 16-\mathrm{C} 17$ & $-17.9(3)$ \\
\hline $\mathrm{C} 3-\mathrm{C} 2-\mathrm{C} 7-\mathrm{Se}$ & $179.15(13)$ & $\mathrm{C} 14-\mathrm{C} 15-\mathrm{C} 16-\mathrm{C} 17$ & $161.00(18)$ \\
\hline $\mathrm{C} 13-\mathrm{C} 8-\mathrm{C} 9-\mathrm{C} 10$ & $-2.4(3)$ & $\mathrm{C} 21-\mathrm{C} 16-\mathrm{C} 17-\mathrm{C} 18$ & $-0.2(3)$ \\
\hline $\mathrm{Se}-\mathrm{C} 8-\mathrm{C} 9-\mathrm{C} 10$ & $173.23(15)$ & $\mathrm{C} 15-\mathrm{C} 16-\mathrm{C} 17-\mathrm{C} 18$ & $-179.43(17)$ \\
\hline $\mathrm{C} 8-\mathrm{C} 9-\mathrm{C} 10-\mathrm{C} 11$ & $0.9(3)$ & $\mathrm{C} 16-\mathrm{C} 17-\mathrm{C} 18-\mathrm{C} 19$ & $0.0(3)$ \\
\hline $\mathrm{C} 9-\mathrm{C} 10-\mathrm{C} 11-\mathrm{C} 12$ & $1.1(3)$ & $\mathrm{C} 17-\mathrm{C} 18-\mathrm{C} 19-\mathrm{C} 20$ & $0.2(3)$ \\
\hline $\mathrm{C} 10-\mathrm{C} 11-\mathrm{C} 12-\mathrm{C} 13$ & $-1.5(3)$ & $\mathrm{C} 18-\mathrm{C} 19-\mathrm{C} 20-\mathrm{C} 21$ & $-0.2(3)$ \\
\hline $\mathrm{C} 11-\mathrm{C} 12-\mathrm{C} 13-\mathrm{C} 8$ & $0.0(3)$ & $\mathrm{C} 19-\mathrm{C} 20-\mathrm{C} 21-\mathrm{C} 16$ & $0.0(3)$ \\
\hline $\mathrm{C} 11-\mathrm{C} 12-\mathrm{C} 13-\mathrm{N} 1$ & $179.48(17)$ & $\mathrm{C} 17-\mathrm{C} 16-\mathrm{C} 21-\mathrm{C} 20$ & $0.2(3)$ \\
\hline $\mathrm{C} 9-\mathrm{C} 8-\mathrm{C} 13-\mathrm{C} 12$ & $1.9(3)$ & $\mathrm{C} 15-\mathrm{C} 16-\mathrm{C} 21-\mathrm{C} 20$ & $179.44(17)$ \\
\hline $\mathrm{Se}-\mathrm{C} 8-\mathrm{C} 13-\mathrm{C} 12$ & $-173.39(15)$ & & \\
\hline
\end{tabular}

Hydrogen-bond geometry $\left(A,{ }^{\circ}\right)$

$\mathrm{Cg} 1$ is the centroid of the $\mathrm{C} 1-\mathrm{C} 6$ ring.

\begin{tabular}{lllll}
\hline$D-\mathrm{H} \cdots A$ & $D-\mathrm{H}$ & $\mathrm{H} \cdots A$ & $D \cdots A$ & $D-\mathrm{H} \cdots A$ \\
\hline $\mathrm{C} 18-\mathrm{H} 18 \cdots \mathrm{O} 1^{\mathrm{i}}$ & 0.95 & 2.54 & $3.472(2)$ & 165 \\
$\mathrm{C} 14-\mathrm{H} 14 \cdots \mathrm{N} 3^{\mathrm{ii}}$ & 0.95 & 2.58 & $3.520(2)$ & 170 \\
$\mathrm{C} 10-\mathrm{H} 10 \cdots C g 1^{\mathrm{iii}}$ & 0.95 & 2.82 & $3.630(2)$ & 144 \\
\hline
\end{tabular}

Symmetry codes: (i) $-x+2,-y+2,-z+1$; (ii) $x-1, y, z$; (iii) $-x+1,-y+1,-z+2$. 